\title{
Randomized, Double-Blind Study of the Safety of the Liquid Versus Lyophilized Formulation of Palivizumab in Premature Infants and Children with Chronic Lung Disease of Prematurity
}

\author{
Doris Makari • Kathryn M. Jensen • Brian Harris • Hasan S. Jafri
}

To view enhanced content go to www.infectiousdiseases-open.com Received: June 30, 2014 / Published online: August 26, 2014

(C) The Author(s) 2014. This article is published with open access at Springerlink.com

\section{ABSTRACT}

Introduction: To avoid the need for reconstitution required by lyophilized palivizumab, a liquid formulation was developed. This study assessed the safety and antidrug antibodies (ADA) of the liquid formulation of palivizumab compared with the lyophilized formulation.

Methods: This phase 4, randomized, doubleblind, multicenter study included children with chronic lung disease of prematurity who were $\leq 24$ months of age and children born prematurely with a gestational age of $\leq 35$ weeks who were $\leq 6$ months of age at randomization. Subjects were randomized 1:1 to $15 \mathrm{mg} / \mathrm{kg}$ of either liquid or lyophilized palivizumab administered via intramuscular injection every 30 days for a total of 5

Electronic supplementary material The online version of this article (doi:10.1007/s40121-014-0033-y) contains supplementary material, which is available to authorized users.

D. Makari · K. M. Jensen · B. Harris · H. S. Jafri ( $₫)$ MedImmune, Gaithersburg, MD, USA

e-mail: jafrih@medimmune.com injections. Safety was assessed based on serious adverse events (SAEs). ADA to palivizumab was assessed using blood collected at baseline and at a time point between study days 240 and 300 .

Results: A total of 413 subjects were included in the analyses. The incidence of SAEs reported was $8.5 \%$ with liquid palivizumab and 5.9\% with lyophilized palivizumab; none were deemed drug-related. The reported SAEs were consistent with expected conditions in this pediatric age group; there was no increase in respiratory syncytial virus (RSV) disease with liquid palivizumab. At study days 240-300, antipalivizumab antibodies were detected in none of the subjects in the liquid palivizumab group and in 1 subject in the lyophilized group. The true ADA percent positive, based on the upper limit of the $95 \%$ confidence interval (CI), was $<1.5 \%$ for both treatments combined.

Conclusion: The frequency of detection of ADAs was low. The true ADA percent positive for both treatment groups combined based on the upper limit of the $95 \%$ CI was $<1.5 \%$. The type and frequency of SAEs reported were as expected, and there was no evidence of an increase in RSV disease with liquid palivizumab. 
Keywords: Antidrug antibody; Children; Premature infants; Chronic lung disease of prematurity; Liquid; Lyophilized; Palivizumab; Respiratory syncytial virus disease; Safety

\section{INTRODUCTION}

Respiratory syncytial virus (RSV) is a major respiratory viral pathogen in infants and young children worldwide; there were approximately 34 million cases of RSVassociated acute lower respiratory tract infection in children $<5$ years of age globally in 2005 [1]. Approximately 10\% of these cases (3.4 million) were severe enough to require hospital admission, and there were approximately 200,000 deaths [1]. Risk factors for severe RSV disease include preterm birth (born at $\leq 35$ weeks gestation), congenital heart disease, and chronic lung disease of prematurity [2].

Respiratory syncytial virus immune globulin intravenous (RSV-IGIV; RespiGam ${ }^{\circledR}$, MedImmune; Gaithersburg, MD, USA) was the first product used for preventing severe RSV disease and required a 4 -h intravenous infusion. Palivizumab (MedImmune), an F-proteinspecific humanized monoclonal antibody, was an advance in RSV prevention, as it could be given intramuscularly [3]. Palivizumab, first licensed in the United States in 1998, is indicated for the prevention of serious lower respiratory tract disease due to RSV in children at high risk of RSV disease [4]. It is currently licensed in more than 80 countries [5]. The efficacy of palivizumab has been established in three randomized, placebo-controlled studies [6-8]. Initially, palivizumab was available only in a lyophilized formulation that required reconstitution with sterile water to a concentration of 50 or $100 \mathrm{mg} / \mathrm{mL}$ [9]. To avoid the need for reconstitution, a liquid formulation of palivizumab was developed [9]. The liquid formulation of palivizumab allows for more simplified administration, which is valuable for healthcare providers and decreases the amount of time high-risk infants must spend in a waiting room with potential exposure to other sick children. Additionally, liquid palivizumab reduces the potential error in amount of medication administered that could occur from improper reconstitution of the medication [10]. After the liquid formulation was assessed for safety in adults [11], a study of children $\leq 6$ months of age with a history of prematurity showed that these 2 formulations of palivizumab were bioequivalent with a similar safety profile, leading to the approval of liquid palivizumab by the US Food and Drug Administration in 2004 [12]. In addition, antidrug antibodies (ADA) can negatively affect the pharmacokinetics and pharmacodynamics of a drug, leading to potential adverse effects or loss of efficacy [13]. This study measured ADA approximately 4-7 months after the last dose of study medication, which had not been previously studied.

This study was a postmarketing commitment and assessed the safety and ADAs of the liquid formulation of palivizumab compared with the lyophilized formulation in children at high risk for the development of serious RSV disease.

\section{METHODS}

\section{Subjects}

The study included medically stable children with chronic lung disease of prematurity who were $\leq 24$ months of age at randomization and 
children born prematurely with a gestational age of $\leq 35$ weeks who were $\leq 6$ months of age at randomization. Children were excluded if they received lyophilized palivizumab, IGIV, or other RSV-specific monoclonal antibody within 3 months before randomization, were receiving mechanical ventilation at the time of study entry, had congenital heart disease, acute respiratory or other acute infection or illness, a life expectancy of $<6$ months, or had a mother diagnosed with human immunodeficiency virus, unless the child had been proven to not be infected.

Study approval was obtained by the individual Institutional Review Boards of some sites, whereas approval was obtained by a centralized Institutional Review Board (Chesapeake IRB, Columbia, MD, USA) for the remaining sites. The study was conducted in accordance with the Declaration of Helsinki. Written informed consent was obtained from each study subject's parent or legal guardian before study entry.

\section{Study Drug}

Study medication was administered via intramuscular injection every 30 days during the RSV season, for a total of 5 injections. All subjects were scheduled to receive 5 injections. Liquid palivizumab was supplied in sterile vials containing $100 \mathrm{mg}$ of palivizumab in $1 \mathrm{~mL}$ of a sterile, preservative-free liquid, formulated with $25 \mathrm{mM}$ histidine and $1.6 \mathrm{mM}$ glycine. Lyophilized palivizumab was supplied in sterile vials containing $100 \mathrm{mg}$ of sterile lyophilized product that when formulated contained $25 \mathrm{mM}$ histidine, $1.6 \mathrm{mM}$ glycine, and 3\% mannitol. Lyophilized palivizumab required reconstitution with $1 \mathrm{~mL}$ of sterile water for injection to yield palivizumab at a concentration of $100 \mathrm{mg} / \mathrm{mL}$. Liquid and lyophilized palivizumab were similar in formulation with the exception of the excipients.

\section{Study Design}

This phase 4, randomized, double-blind, multicenter study enrolled subjects over 2 RSV seasons (ClinicalTrials.gov \#NCT00233064) from October 2005 to October 2007 across 51 sites in the United States. Subjects were randomized $1: 1$ to $15 \mathrm{mg} / \mathrm{kg}$ of palivizumab liquid or lyophilized formulation.

The study was conducted in a double-blind manner with the medical monitor, statistician, project management, site monitors, data management, subjects' parents, and the clinical site staff blinded to study treatment assignment throughout the study. An independent monitor who only received pharmacy records and the investigational agent manager at the study site were the only people with access to information that identified a subject's treatment allocation. Neither individual was to reveal to anyone the treatment arm to which a subject was assigned.

The study drug was supplied to the pharmacy as open-label vials of liquid or lyophilized palivizumab. The investigational agent manager prepared the study drug and dispensed it in identically appearing syringes, labeled using the subjects' initials.

\section{Safety}

Safety was assessed based on serious adverse events (SAEs). Subjects were monitored through study day 150 or until the resolution of any serious events, whichever was longer. SAEs were defined as those that resulted in death, were life-threatening, led to hospitalization, or prolongation of an existing hospitalization. 
SAEs were graded by severity (mild, moderate, severe, or life-threatening) and by relationship to study drug (none, remote, possible, probable, or definite) as determined by the principal investigator.

\section{Antidrug Antibodies}

ADA to palivizumab was assessed using blood collected at baseline before the first dose of study medication and at 1 time point between study days 240 and 300 (approximately 4-7 months after the last dose of study medication). Antipalivizumab antibody analyses were performed at PPD (Richmond, VA, USA) using a validated enzyme-linked immunosorbent assay (ELISA) (developed by MedImmune) [14]. Controls were prepared by adding a goat polyclonal antipalivizumab antibody into normal human serum. Duplicate determinations of the positive control and negative controls were performed. Results were analyzed using Softmax Pro 4.8 software (Molecular Devices Corporation. Sunnyvale, CA, USA). Positive unknown samples were titered on a second plate beginning with the original sample and diluting 1:2 with normal human serum across the plate in duplicate. The endpoint titer was defined as the highest serum dilution tested that showed a positive response. The lowest dilution value for which a positive result could be obtained was 1:10 because all samples were diluted 1:10 before performing the assay.

\section{Statistical Analyses}

The safety analysis included all subjects who received any study medication. The ADA analysis included subjects who received $\geq 2$ doses of study medication and had $\geq 1$ blood sample collected. The sample size calculation was based on findings from the pivotal phase 3 study [6], which showed approximately $1 \%$ detection of ADA after a single season of dosing with lyophilized palivizumab. A sample size of 200 per treatment group was chosen so that the upper limit of the $95 \%$ confidence interval (CI) would be below $3 \%$.

Categorical data were summarized by the number and percent of subjects in each category. To determine the percent positive ADA, 95\% CIs were also calculated. No formal tests of comparison were planned or conducted. Statistical analyses were conducted using the SAS $^{\circledR}$ System software version 6.12 and/or 8.2 for Windows, or higher (SAS Institute, Inc., Cary, NC, USA).

\section{RESULTS}

\section{Subjects}

A total of 417 subjects were randomized into the study across the 51 sites in the United States, and 413 subjects were followed and included in all of the safety analyses; 4 subjects from 1 site were excluded from the analyses because the site did not provide any additional information or data regarding the subjects other than the fact that they received all 5 doses of palivizumab. Of the 413 subjects, 211 were randomized into the liquid palivizumab group and 202 were randomized into the lyophilized palivizumab group. Overall, 26/413 (6.3\%) subjects did not complete the study. The most common reasons for not completing the study were lost to follow-up [14/413 (3.4\%)] and withdrawal of consent [8/413 (1.9\%); Fig. 1]. Demographic and baseline characteristics, including age, gender, race, and weight, were similar between the liquid and lyophilized palivizumab groups (Table 1). 


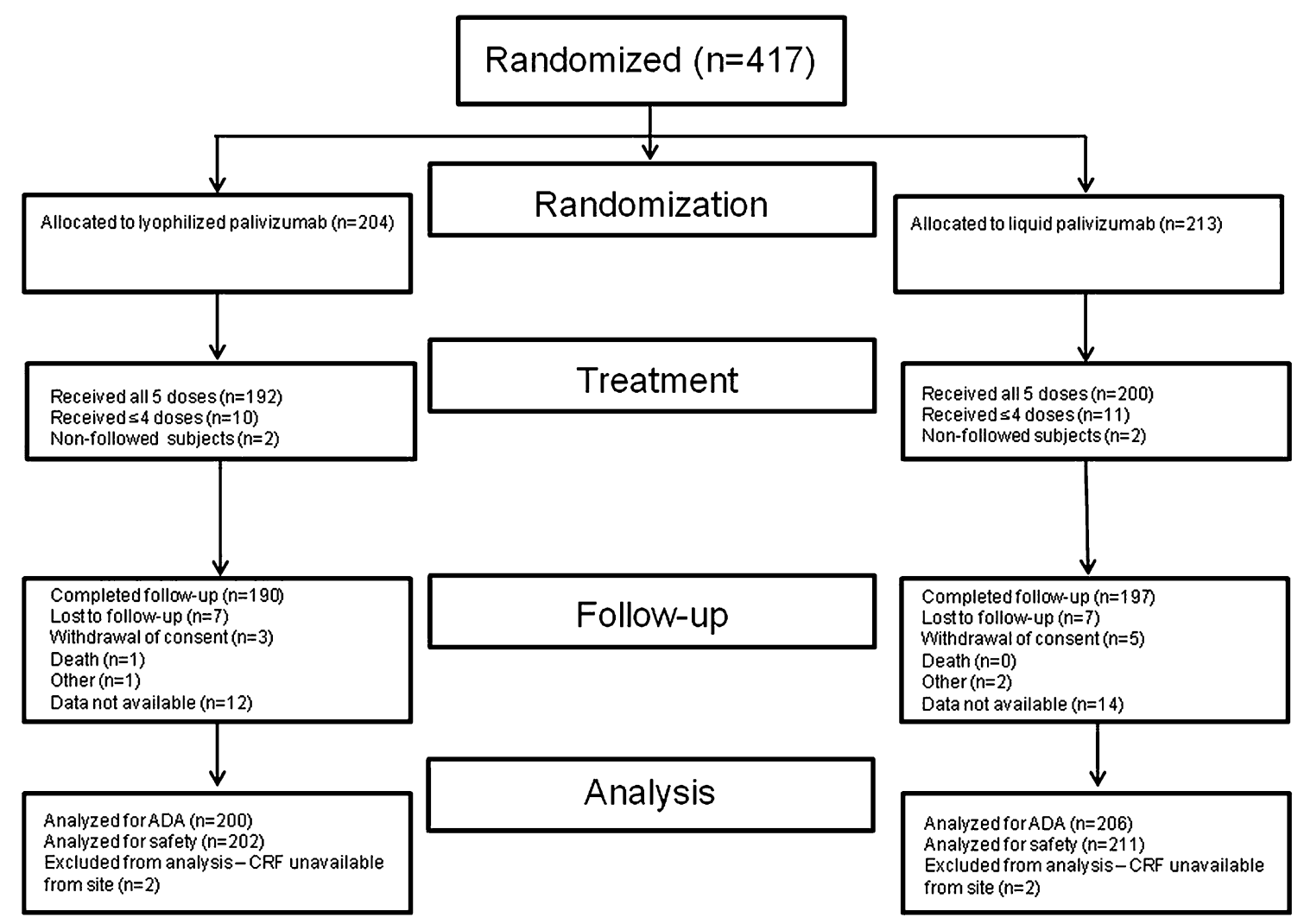

Fig. 1 Disposition of subjects. $A D A$ Antidrug antibody, $C R F$ case report form

Safety

The majority of subjects in both study groups received all 5 doses of medication [94.8\% (200/ 211) in the liquid palivizumab group and $95 \%$ $(192 / 202)$ in the lyophilized palivizumab group]. The incidence of SAEs reported was $8.5 \%(18 / 211)$ with liquid palivizumab and $5.9 \%(12 / 202)$ with lyophilized palivizumab (Table 2). The reported SAEs were consistent with common conditions in this pediatric age group. The most common SAEs (i.e., those occurring in $\geq 2$ subjects) were bronchiolitis, gastroenteritis, respiratory distress, viral infection, cleft lip, and inguinal hernia (Table 2). The incidence of bronchiolitis was $2.8 \%(6 / 211)$ in the liquid palivizumab group and $1.5 \%(3 / 202)$ in the lyophilized palivizumab group. One subject in the lyophilized palivizumab group died of asphyxia during the study, but the death was deemed not related to the study medication by the study investigator. None of the SAEs were determined by the investigators to be related to study medication.

By system organ class, 2 system organ classes had $>1$ percentage point difference between treatment groups: infections and infestations [liquid, 6.2\% (95\% CI 3.3\%, 10.3\%); lyophilized $3.0 \%(1.1 \%, 6.42 \%)]$ and respiratory, thoracic and mediastinal disorders [liquid, $0.0 \%(0.0 \%$, 1.7\%); lyophilized, $2.0 \%(0.5 \%, 5.0 \%)]$. For infections and infestations, SAEs that may have contributed to a higher incidence in the liquid palivizumab group included bronchiolitis and viral infection. There was no evidence of an 
Table 1 Subject demographics and baseline characteristics

\begin{tabular}{|c|c|c|c|}
\hline Characteristic & $\begin{array}{l}\text { Lyophilized palivizumab } \\
(n=202)\end{array}$ & $\begin{array}{l}\text { Liquid palivizumab } \\
(n=211)\end{array}$ & Total $(n=413)$ \\
\hline \multicolumn{4}{|l|}{ Age (months) } \\
\hline Mean (SD) & $4.0(4.2)$ & $4.6(4.5)$ & $4.3(4.3)$ \\
\hline Median & 2.9 & 3.4 & 3.2 \\
\hline Range & $0.2-22.9$ & $0.2-23.6$ & $0.2-23.6$ \\
\hline \multicolumn{4}{|l|}{ Gestational age (weeks) } \\
\hline Mean (SD) & $32.7(2.5)$ & $32.4(2.7)$ & $32.5(2.6)$ \\
\hline Median & 34.0 & 33.0 & 34.0 \\
\hline Range & $24-36$ & $24-38$ & $24-38$ \\
\hline \multicolumn{4}{|l|}{ Gender, $n(\%)$} \\
\hline Male & $103(51.0)$ & $107(50.7)$ & $210(50.8)$ \\
\hline \multicolumn{4}{|l|}{ Race, $n(\%)$} \\
\hline White/non-Hispanic & $149(73.8)$ & $151(71.6)$ & $300(72.6)$ \\
\hline Black & $24(11.9)$ & $25(11.8)$ & $49(11.9)$ \\
\hline Hispanic & $14(6.9)$ & $22(10.4)$ & $36(8.7)$ \\
\hline Asian & $3(1.5)$ & $1(0.5)$ & $4(1.0)$ \\
\hline Other & $12(5.9)$ & $12(5.7)$ & $24(5.8)$ \\
\hline \multicolumn{4}{|l|}{ Weight at day $0(\mathrm{~kg})$} \\
\hline Mean (SD) & $5.1(2.3)$ & $5.3(2.3)$ & $5.2(2.3)$ \\
\hline Median & 4.74 & 5.20 & 5.00 \\
\hline Range & $1.8-13.8$ & $1.8-14.5$ & $1.8-14.5$ \\
\hline \multicolumn{4}{|c|}{ CLD of prematurity, $n(\%)$} \\
\hline Yes & $26(12.9)$ & $35(16.6)$ & $61(14.8)$ \\
\hline
\end{tabular}

$C L D$ Chronic lung disease, $S D$ standard deviation

increase in RSV disease with liquid palivizumab. Of the 9 events of bronchiolitis, 7 were tested locally for RSV (liquid, $n=5$; lyophilized, $n=2$ ) and all 7 were negative. A single event of bronchopneumonia (in the liquid palivizumab group) was tested locally and was negative for RSV. Both events of viral infection were negative for RSV based on local testing. The events of respiratory, thoracic and mediastinal disorders reported in the lyophilized palivizumab group were respiratory distress (2 subjects), and apnea, asphyxia, and dyspnea (each in 1 subject). The SAE of asphyxia resulted in death (described above). The remaining events occurred sporadically throughout dosing; all required hospitalization and resolved within 2-10 days after treatment. The events of apnea, dyspnea, and asphyxia were tested locally for RSV and all were negative. 
Table 2 Serious adverse events

\begin{tabular}{|c|c|c|c|}
\hline SAE, $n(\%)$ & $\begin{array}{l}\text { Lyophilized palivizumab } \\
(n=202)\end{array}$ & $\begin{array}{l}\text { Liquid palivizumab } \\
(n=211)\end{array}$ & $\begin{array}{l}\text { Total } \\
(n=413)\end{array}$ \\
\hline $\begin{array}{l}\text { Total number of subjects } \\
\text { reporting } \geq 1 \text { SAE }\end{array}$ & $12(5.9)$ & $18(8.5)$ & $30(7.3)$ \\
\hline Bronchiolitis & $3(1.5)$ & $6(2.8)$ & $9(2.2)$ \\
\hline Gastroenteritis & $2(1.0)$ & $2(0.9)$ & $4(1.0)$ \\
\hline Respiratory distress & $2(1.0)$ & $0(0.0)$ & $2(0.5)$ \\
\hline Viral infection & $0(0.0)$ & $2(0.9)$ & $2(0.5)$ \\
\hline Cleft lip & $1(0.5)$ & $1(0.5)$ & $2(0.5)$ \\
\hline Inguinal hernia & $1(0.5)$ & $1(0.5)$ & $2(0.5)$ \\
\hline Abscess & $1(0.5)$ & $0(0.0)$ & $1(0.2)$ \\
\hline Anal fissure & $0(0.0)$ & $1(0.5)$ & $1(0.2)$ \\
\hline Apnea & $1(0.5)$ & $0(0.0)$ & $1(0.2)$ \\
\hline Asphyxia & $1(0.5)$ & $0(0.0)$ & $1(0.2)$ \\
\hline Bronchopneumonia & $0(0.0)$ & $1(0.5)$ & $1(0.2)$ \\
\hline Cellulitis & $0(0.0)$ & $1(0.5)$ & $1(0.2)$ \\
\hline Complex partial seizures & $0(0.0)$ & $1(0.5)$ & $1(0.2)$ \\
\hline Convulsions & $0(0.0)$ & $1(0.5)$ & $1(0.2)$ \\
\hline Craniosynostosis & $0(0.0)$ & $1(0.5)$ & $1(0.2)$ \\
\hline Dehydration & $0(0.0)$ & $1(0.5)$ & $1(0.2)$ \\
\hline Dyspnea & $1(0.5)$ & $0(0.0)$ & $1(0.2)$ \\
\hline Failure to thrive & $1(0.5)$ & $0(0.0)$ & $1(0.2)$ \\
\hline Gastroenteritis rotavirus & $0(0.0)$ & $1(0.5)$ & $1(0.2)$ \\
\hline Gastroesophageal reflux disease & $0(0.0)$ & $1(0.5)$ & $1(0.2)$ \\
\hline Hydronephrosis & $0(0.0)$ & $1(0.5)$ & $1(0.2)$ \\
\hline Infectious croup & $0(0.0)$ & $1(0.5)$ & $1(0.2)$ \\
\hline Lymphadenitis & $0(0.0)$ & $1(0.5)$ & $1(0.2)$ \\
\hline Occult blood positive & $1(0.5)$ & $0(0.0)$ & $1(0.2)$ \\
\hline Umbilical hernia & $0(0.0)$ & $1(0.5)$ & $1(0.2)$ \\
\hline
\end{tabular}

SAE Serious adverse event

\section{Antidrug Antibodies}

At baseline, none of the subjects exhibited antipalivizumab antibodies. From study days
240-300, antipalivizumab antibodies were detected in none of the subjects in the liquid palivizumab group and in $1 / 188$ subject (0.5\%) in the lyophilized palivizumab group (at 
154 days post final dose), with an overall percent positive of $0.3 \%$ (1/379) for both treatment groups combined. Given these observations and the number of subjects studied, the true ADA percent positive, based on the upper limit of the exact $95 \%$ CI, is at most $1.9 \%$ for the liquid palivizumab group, $2.9 \%$ for the lyophilized palivizumab group, and $1.5 \%$ for both treatments combined.

\section{DISCUSSION}

Liquid palivizumab was developed to avoid the need for reconstitution required by lyophilized palivizumab. Since 2006, liquid palivizumab has been the only formulation distributed in the United States, and is estimated to have been administered to one million infants [15]. Findings from this study of children at high risk for serious RSV disease showed that liquid and lyophilized formulations exhibit a comparable safety profile with similar reported SAEs.

The present safety findings generally are consistent with findings from a randomized, double-blind, cross-over study of infants aged $\leq 6$ months who were born $\leq 35$ weeks gestational age [12]. In that study, the percentages of infants with SAEs were similar (liquid, 3.3\%; lyophilized, 2.6\%) [12]. The type and frequency of SAEs reported were similar between the liquid and lyophilized palivizumab groups [12]. The incidence of bronchiolitis SAE reported was also similar between the 2 groups (liquid, 2.8\%; lyophilized, 1.5\%).

The ADA results in the present and previous studies were based on positive palivizumab antibodies using an ELISA, which is limited in its ability to detect antipalivizumab antibodies in the presence of palivizumab [4]. In the present study, the true ADA percent positive for both treatment groups combined based on the upper limit of the 95\% CI was at most 1.5\%. The $0.5 \%$ observed ADA percent positive (with an upper limit of the $95 \%$ CI of $2.9 \%$ ) for lyophilized palivizumab reported in the present study was consistent with the $1.2 \%$ observed ADA percent positive reported in a previous phase 3 trial of lyophilized palivizumab in 1,502 children [6]. In another previous trial of highrisk preterm children $\leq 24$ months of age, the ADA percent positive was $0.3 \%$ for both palivizumab formulations combined [4]. It is possible that the ADA percent positive in both study arms of the present study could have been higher had the current drug-tolerant electrochemiluminescence (ECLA) assay been used. However, 2 studies of liquid palivizumab recipients that used an ECLA to determine ADA also demonstrated ADA percents positive of $1.1 \%$ and $1.5 \%$ [4].

\section{ACKNOWLEDGMENTS}

This study and article publication charges were funded by MedImmune. Medical writing and editorial assistance, provided by John E. Fincke, Ph.D., and Anny Wu, Pharm.D., of Complete Healthcare Communications, Inc. (Chadds Ford, PA, USA), was supported by MedImmune. All named authors meet the ICMJE criteria for authorship for this manuscript, take responsibility for the integrity of the work as a whole, and have given final approval to the version to be published. All authors had full access to all of the data in this study and take complete responsibility for the integrity of the data and accuracy of the data analysis.

Conflict of interest. Doris Makari is an employee of MedImmune and may have stock 
or stock options in AstraZeneca, the parent company of MedImmune. Kathryn M. Jensen is an employee of MedImmune and may have stock or stock options in AstraZeneca, the parent company of MedImmune. Brian Harris is an employee of MedImmune and may have stock or stock options in AstraZeneca, the parent company of MedImmune. Hasan S. Jafri is an employee of MedImmune and may have stock or stock options in AstraZeneca, the parent company of MedImmune.

Compliance with ethics guidelines. All study procedures followed were in accordance with the ethical standards of the responsible committee on human experimentation (institutional and national) and with the Helsinki Declaration of 1975 , as revised in 2000 and 2008. Informed consent was obtained from all patients for being included in the study.

Open Access. This article is distributed under the terms of the Creative Commons Attribution Noncommercial License which permits any noncommercial use, distribution, and reproduction in any medium, provided the original author(s) and the source are credited.

\section{REFERENCES}

1. Nair H, Nokes DJ, Gessner BD, et al. Global burden of acute lower respiratory infections due to respiratory syncytial virus in young children: a systematic review and meta-analysis. Lancet. 2010;375:1545-55.

2. American Academy of Pediatrics. Policy statement-modified recommendations for use of palivizumab for prevention of respiratory syncytial virus infections. Pediatrics. 2009;124:1694-1701.

3. Johnson S, Oliver C, Prince GA, et al. Development of a humanized monoclonal antibody (MEDI-493) with potent in vitro and in vivo activity against respiratory syncytial virus. J Infect Dis. 1997;176:1215-24.

4. Palivizumab. Full prescribing information. Gaithersburg: MedImmune; 2014.

5. La Via WV, Notario GF, Yu XQ, et al. Three monthly doses of palivizumab are not adequate for 5-month protection: a population pharmacokinetic analysis. Pulm Pharmacol Ther. 2013;26:666-71.

6. The IMpact-RSV Study Group. Palivizumab, a humanized respiratory syncytial virus monoclonal antibody, reduces hospitalization from respiratory syncytial virus infection in high-risk infants. Pediatrics. 1998;102:531-7.

7. Blanken MO, Rovers MM, Molenaar JM, et al. Respiratory syncytial virus and recurrent wheeze in healthy preterm infants. $\mathrm{N}$ Engl $\mathrm{J}$ Med. 2013;368:1791-9.

8. Feltes TF, Cabalka AK, Meissner $\mathrm{HC}$, et al. Palivizumab prophylaxis reduces hospitalization due to respiratory syncytial virus in young children with hemodynamically significant congenital heart disease. J Pediatr. 2003;143: 532-40.

9. Mejias A, Chavez-Bueno S, Sanchez PJ. Respiratory syncytial virus prophylaxis. Neoreviews. 2005;6:e26-31.

10. ASHP guidelines on preventing medication errors in hospitals. Am J Hosp Pharm. 1993;50:305-14.

11. Data on File-Study MI-CP080. Gaithersburg: MedImmune, LLC.

12. Data on File-Study MI-CP097. A phase 2, randomized, double-blind, two-period, cross-over study to evaluate the pharmacokinetics, safety and tolerability of a liquid formulation of palivizumab (MEDI-493, Synagis ${ }^{\circledR}$ ), a humanized respiratory syncytial virus monoclonal antibody, in children with a history of prematurity. Gaithersburg: MedImmune, LLC; 2007.

13. Gupta S, Devanarayan V, Finco D, et al. Recommendations for the validation of cell-based assays used for the detection of neutralizing antibody immune responses elicited against biological therapeutics. J Pharm Biomed Anal. 2011;55:878-88.

14. Carbonell-Estrany X, Simoes EA, Dagan R, et al. Motavizumab for prophylaxis of respiratory syncytial virus in high-risk children: a noninferiority trial. Pediatrics. 2010;125:e35-51.

15. Data on File. Gaithersburg: MedImmune. 